\title{
An Event-Based Service Framework for Learning, Querying, and Monitoring Multivariate Time Series
}

\author{
Chun-Kit Ngan ${ }^{1}$, Alexander Brodsky ${ }^{1}$, and Jessica Lin ${ }^{1}$, \\ ${ }^{1}$ Department of Computer Science, George Mason University, 4400 University Drive MSN \\ 4A5, Fairfax, Virginia 22030-4422, United States \\ \{cngan, brodsky, jessica\}@gmu.edu
}

\begin{abstract}
We propose an event-based service framework for Multivariate Time Series Analytics (MTSA) that supports model definition, querying, parameter learning, model evaluation, monitoring, and decision recommendation on events. Our approach combines the strengths of both domain-knowledge-based and formal-learning-based approaches for maximizing utility on events over multivariate time series. More specifically, we identify multivariate time series parametric estimation problems, in which the objective function is dependent on the time points from which the parameters are learned. We propose an algorithm that guarantees to find the optimal time point(s), and we show that our approach produces results that are superior to those of the domainknowledge-based approach and the logit regression model. We also develop MTSA data model and query language for the services of parameter learning, querying, and monitoring.
\end{abstract}

Keywords: Event-Based Service Framework, Multivariate Time Series, Parameter Learning, Decision Support

\section{Introduction}

Making decisions over multivariate time series is an important topic which has gained significant interest in the past decade, as two or more time series are often observed simultaneously in many fields. In business and economics, financial analysts and researchers monitor daily stock prices, weekly interest rates, and monthly price indices to analyze different states of stock markets. In medical studies, physicians and scientists measure patients' diastolic and systolic blood pressure over time and electrocardiogram tracings to evaluate the patients' health of respiratory systems. In social sciences, sociologists and demographers study annual birth rates, mortality rates, accident rates, and various crime rates to dig out hidden social problems within a community. The purpose of these measures over multivariate time series is to assist the specialists in understanding the same problem in different perspectives within particular domains. For those significant events to be identified and detected over multivariate time series, the events can lead the professionals to make better decisions and take more reasonable actions promptly. Those events may include index bottoms and tops in financial markets, irregular readings on blood pressure and pulse anomalies on electrocardiogram, as well as low birth but high death rates in a 
population region. To support such event-based decision-making and determination over multivariate time series, we propose a service framework, Multivariate Time Series Analytics (MTSA), which consists of services for model definition, querying, parameter learning, model evaluation, monitoring, and decision recommendation on events. Our technical focus of this work is on the problem of event detection; namely, the parameter learning, data monitoring, and decision recommendation services.

Currently, existing approaches to identifying and detecting those interesting events can be roughly divided into two categories: domain-knowledge-based and formallearning-based. The former relies solely on domain expert knowledge. Based on their knowledge and experiences, domain experts determine the conditions that trigger the events of interest. Consider one particular example of the timely event detection of certain conditions in the stock market, e.g., the bear market bottom, that can provide investors a valuable insight into the best investment opportunity. Such identification and detection can aid in the task of decision-making and the determination of action plans. To assist users in making better decisions and determinations, domain experts have identified a set of financial indices that can be used to determine the event, i.e., the bear market bottom or the "best buy" opportunity. The indices include the S\&P 500 percentage decline (SPD), Coppock Guide (CG), Consumer Confidence point drop (CCD), ISM Manufacturing Survey (ISM), and Negative Leadership Composite "Distribution" (NLCD) [1]. If these indices satisfy the pre-defined, parameterized conditions, e.g., SPD $<-20 \%, \mathrm{CG}<0$, etc., [1], it signals that the best period for the investors to buy the stocks is approaching. Often these parameters may reflect some realities since they are set by the domain experts based on their past experiences, observations, intuitions, and domain knowledge. However, they are not always accurate. In addition, the parameters are static, but the problem that we deal with is often dynamic in nature. The market is constantly impacted by many unknown and uncontrollable factors from the business surroundings. Thus, this approach lacks a formal mathematical computation that dynamically learns the parameters to meet the needs of the changing environment.

An alternative approach is to utilize formal learning methods such as non-linear logit regression models $[2,3,4]$. The logit regression models are used to predict the occurrence of an event ( 0 or 1 ) by learning parametric coefficients of the logistic distribution function of the explanatory variables. This is done based on the historical data by applying nonlinear regression models and Maximum Likelihood Estimation (MLE). The main challenge concerning using formal learning methods to support decision-making is that they do not always produce satisfactory results, as they do not consider incorporating domain knowledge into their formal learning approaches. Without domain experts' knowledge, formal learning methods become computationally intensive and time consuming. The whole model building is an iterative and interactive process, including model formulation, parameter estimation, and model evaluation. Despite enormous improvements in computer software in recent years, fitting such nonlinear quantitative decision model is not a trivial task, especially if the parameter learning process involves multiple explanatory variables, i.e., high dimensionality. Working with high-dimensional data creates difficult challenges, a phenomenon known as the "curse of dimensionality". Specifically, the amount of observations required in order to obtain good estimates increases exponentially with the increase of dimensionality. In addition, many learning 
algorithms do not scale well on high dimensional data due to the high computational cost. The parameter computations by formal-learning-based approaches, e.g., logit regression model, are complicated and costly, and they lack the consideration of integrating experts' domain knowledge into the learning process - a step that could potentially reduce the dimensionality. Clearly, both approaches, domain-knowledgebased and formal-learning-based, do not take advantage of each other to learn the optimal decision parameters, which are then used to monitor the events and make better recommendations.

To mitigate the shortcomings of the existing approaches, the proposed MTSA service framework combines the strengths of both the domain-knowledge-based and the formal-learning-based approaches. The service framework supports quick implementation of services towards decision recommendation on events over multivariate time series. More specifically, the MTSA Model Definition Service takes the template of conditions, which determines the occurrence of an event, identified by domain experts - such template consists of inequalities of values in the time sequences - and the Learning Service "parameterizes" it, e.g., SPD $<p_{l}$. The goal of the learning service is to efficiently learn parameters that maximize the objective function, e.g., earnings in our financial example. The Monitoring and Recommendation Service continuously monitors the data stream for data that satisfy the parameterized conditions, in which the parameters have been instantiated by the learning service. We also propose an extension of the relational database model and SQL with highlevel MTSA constructs to support querying, monitoring, and parameter learning.

To this end, we identify multivariate time series parametric estimation problems, in which the objective function is dependent on the time points from which the parameters are learned. With the potentially large data size and multiple variables, classic branch-and-bound approaches have exponential complexity in the worst-case scenario. We develop a new algorithm that guarantees a true optimal time point, with complexity of $O(k N \log N)$, where $\mathrm{N}$ is the size of the learning data set, and $\mathrm{k}$ is the number of parametric time series. To demonstrate the effectiveness and the efficiency of our algorithm, we compare our method with the domain-knowledge-based approach and the logit regression model. As a proof of concept, we conduct an experiment in the financial domain, but note that our framework is applicable to solve problems in different domains. We show that our algorithm is more effective and produces results that are superior to those of the two approaches mentioned above. More specifically, in our experiments we show that our algorithm outperforms the financial experts' recommendation and the logit regression model, resulting in higher earnings for our imaginary investor.

The rest of the paper is organized as follows. In Section 2, we provide an overview on the MTSA service framework. We discuss the learning and monitoring services by defining the Expert Query Parametric Estimation (EQPE) model in Section 3. Section 4 explains the domain-knowledge-inspired learning algorithm and shows the experimental evaluation on stock market data. In Section 5, we describe the MTSA data model and query language. Section 6 contains the conclusions and future work. 


\section{An Event-Based Service Framework for Multivariate Time Series Analytics (MTSA)}

Fig. 1 shows a range of common services that is desirable to be offered over the internet. The MTSA Model Definition Service provides a parametric model template, which determines the occurrence of an event, identified by the domain experts. In the financial example that predicts the event, i.e., market bottom, the model template may consist of indices such as S\&P 500 percentage decline (SPD), Coppock Guide (CG), etc. These indices are associated with their respective inequality constraints, for example, SPD $<p_{1}$ and $\mathrm{CG}<p_{2}$. Given such a parametric model template in a given domain, the Monitoring and Recommendation Service continuously screens the incoming data stream for indices that satisfy all the constraints which specify when the event of interest, e.g., the market bottom, has occurred, and recommends an action, e.g., buying stock. Note that in the traditional approach, the decision parameters $p_{1}$ and $p_{2}$ are specified by the domain experts, e.g., SPD $<-20 \%$ and CG $<0$. However, using such hard-set parameters cannot capture the dynamics of the rapidly changing market. The Parameter Learning Service parameterizes the template, e.g., SPD $<p_{I}$ and $\mathrm{CG}<p_{2}$, and supports learning of the parameters from the historic time series. The accuracy of the decision parameters are ensured through the Model Accuracy and Quality Evaluation Service, which validates the prediction, i.e., market bottom, with the observed real data, and updates the model if necessary. The Querying Service allows the service developers and database programmers to express the complex information services over multivariate time series mentioned above in a high-level abstraction.

The event-based service framework for multivariate time series analytics (MTSA) provides a medium that supports quick implementation of the services described above. The MTSA service framework is illustrated in Fig. 2. It consists of three layers: data integration, information processing, and query language. The top layer is the MTSA Model Definition and Query Language, which extends the relational database model with time series and events, and SQL with MTSA constructs. The middle layer supports the MTSA constructs including MTSA model template definition, querying, parameter learning, model evaluation, data monitoring, and decision recommendation. The bottom, Data Integration Layer, allows service providers to interact with external data services and collect time series data from heterogeneous sources, as well as from local repositories. This integration layer provides a concentric view of the collected data. The integration of the model template and the learned parameters, which may be available both locally and through external services, is also supported by the Data Integration Layer. 


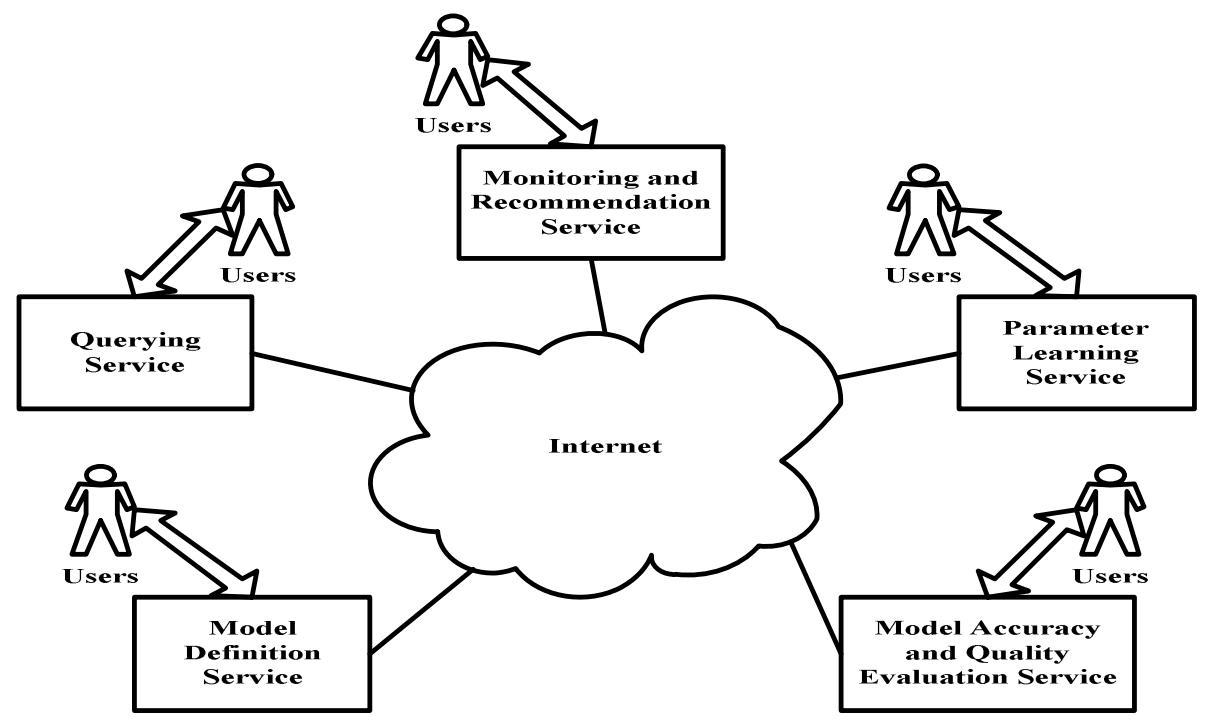

Fig. 1. Services for Multivariate Time Series Over Internet.

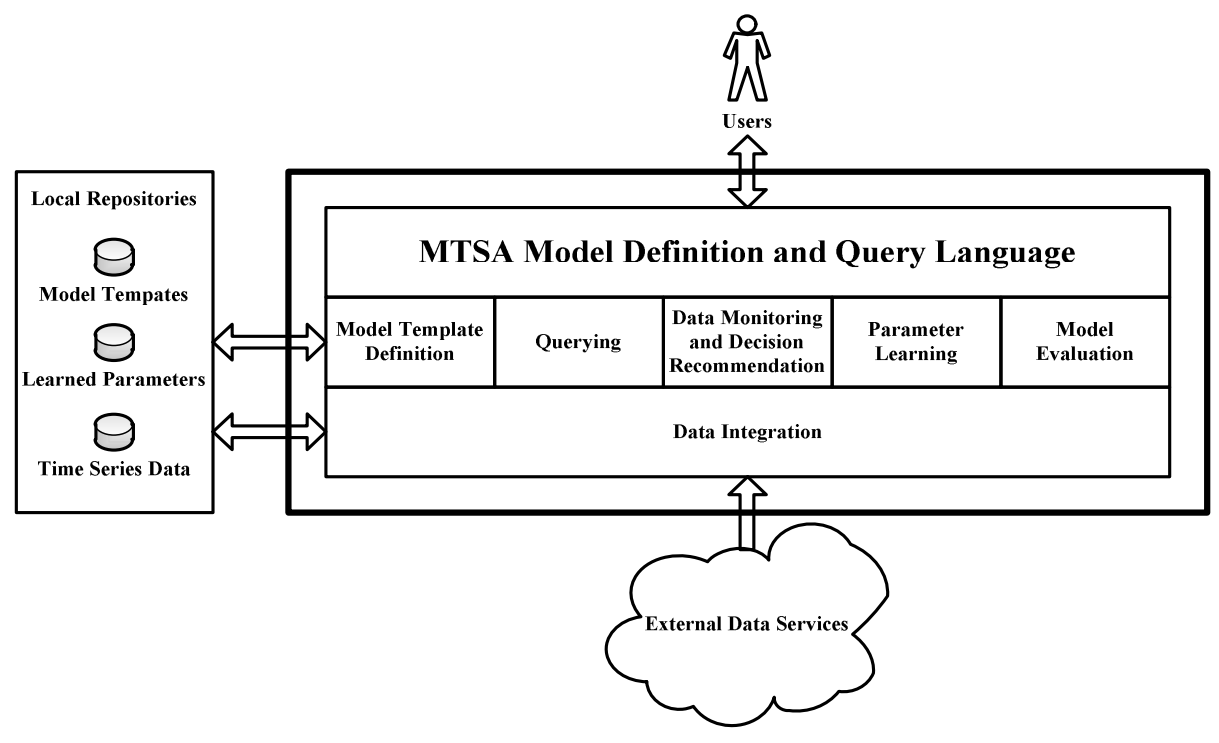

Fig. 2. An Event-Based Service Framework for Multivariate Time Series Analytics. 


\section{Expert Query Parametric Estimation (EQPE) Model}

In this section, we discuss in detail the methodologies used in the Parameter Learning Service and the Monitoring and Recommendation Service in the MTSA framework. More specifically, we review the mathematical formulations of the Expert Query Parametric Estimation (EQPE) problem and solution. We also use the examples to explain them in detail.

The goal of an EQPE problem is to find optimal values of decision parameters that maximize an objective function over historical, multivariate time series. For an EQPE problem being constructed, we need to define a set of mathematical notations and a model for it. We assume that the time domain $\mathbf{T}$ is represented by a set of natural numbers: $\mathbf{T}=\mathbf{N}$, and that we are also given a vector of $\mathrm{n}$ real-valued parameter variables $\left(p_{1}, p_{2}, \ldots, p_{n}\right)$.

Definition 1. Time Series: A time series $S$ is a function $S: T \rightarrow R$, where $T$ is the time domain, and $\mathrm{R}$ is the set of real numbers.

Definition 2. Parametric Monitoring Constraint: A parametric monitoring constraint $\mathrm{C}\left(S_{l}(t), S_{2}(t), \ldots, S_{k}(t), p_{l}, p_{2}, \ldots, p_{n}\right)$ is a symbolic expression in terms of $S_{l}(t), S_{2}(t), \ldots$, $S_{k}(t), p_{1}, p_{2}, \ldots, p_{n}$, where $S_{l}(t), S_{2}(t), \ldots, S_{k}(t)$ are time series, $t \in \mathrm{T}$ is a time point, and $\left(p_{1}, p_{2}, \ldots, p_{n}\right)$ is a vector of parameters.

We assume a constraint $C$ written in a language that has the truth-value interpretation I: $R^{k} \times R^{n} \rightarrow$ \{True, False $\}$, i.e., $\mathrm{I}\left(\mathrm{C}\left(S_{l}(t), S_{2}(t), \ldots, S_{k}(t), p_{1}, p_{2}, \ldots, p_{n}\right)\right)=$ True if and only if the constraint $\mathrm{C}$ is satisfied at the time point $t \in \mathrm{T}$ and with the parameters $\left(p_{1}, p_{2}, \ldots, p_{n}\right) \in R^{n}$. In this paper, we focus on conjunctions of inequality constraints: $\mathrm{C}\left(S_{l}(t), S_{2}(t), \ldots, S_{k}(t), p_{1}, p_{2}, \ldots, p_{n}\right)=\Lambda_{\mathrm{i}}\left(S_{i}(t)\right.$ op $\left.p_{j}\right)$, where op $\in\{<, \leq,=$, $\geq,>\}$.

Definition 3. Time Utility Function: A time utility function $\mathrm{U}$ is a function $\mathrm{U}: \mathrm{T} \rightarrow \mathrm{R}$.

Definition 4. Objective Function: Given a time utility function $\mathrm{U}: \mathrm{T} \rightarrow \mathrm{R}$ and a parametric constraint $\mathrm{C}$, an objective function $\mathrm{O}$ is a function $\mathrm{O}: R^{n} \rightarrow R$, which maps a vector of n parameters on $R^{n}$ to a real value $R$, defined as follows. For $\left(p_{1}, p_{2}, \ldots, p_{n}\right)$ $\in R^{n}, \mathrm{O}\left(p_{1}, p_{2}, \ldots, p_{n}\right) \stackrel{\text { def }}{=} \mathrm{U}(t)$, where $\mathrm{U}$ is the utility function, and $\mathrm{t} \in \mathrm{T}$ is the earliest time point that satisfies $\mathrm{C}$, i.e.,

(1) $S_{1}(t) o p_{1} p_{1} \wedge S_{2}(t) o p_{2} p_{2} \wedge \ldots \wedge S_{n}(t) o p_{n} p_{n}$ is satisfied, and

(2) There does not exist $O \leq t^{\prime}<t$, such that $S_{I}\left(t^{\prime}\right) o p_{1} p_{1} \wedge S_{2}\left(t^{\prime}\right) o p_{2} p_{2} \wedge \ldots \wedge S_{n}\left(t^{\prime}\right)$ $o p_{n} p_{n}$ is satisfied.

Definition 5. Expert Query Parametric Estimation (EQPE) Problem: An EQPE problem is a tuple $\langle\boldsymbol{S}, \boldsymbol{P}, \boldsymbol{C}, \boldsymbol{U}\rangle$, where $\boldsymbol{S}=\left\{S_{1}, S_{2} \ldots, S_{k}\right\}$ is a set of k time series, $\boldsymbol{P}=$ $\left\{p_{l}, p_{2}, \ldots, p_{n}\right\}$ is a set of $\mathrm{n}$ real-value parameter variables, $\boldsymbol{C}$ is a parametric constraint in $\boldsymbol{S}$ and $\boldsymbol{P}$, and $\boldsymbol{U}$ is a time utility function.

Intuitively, a solution to an EQPE problem is an instantiation of values into the vector $\boldsymbol{P}$ of $\mathrm{n}$ real-value parameters that maximizes the objective $\boldsymbol{O}$.

Definition 6. Expert Query Parametric Estimation (EQPE) Solution: A solution to the EQPE problem $<\boldsymbol{S}, \boldsymbol{P}, \boldsymbol{C}, \boldsymbol{U}>$ is $\operatorname{argmax} \boldsymbol{O}\left(p_{1}, p_{2}, \ldots, p_{n}\right)$, i.e., the (estimated) values of parameters, $p_{1}, p_{2}, \ldots, p_{n}$, that maximize $\boldsymbol{O}$, where $\boldsymbol{O}$ is the objective function corresponding to $\boldsymbol{U}$.

The base time series in our financial example are shown in Table 1. We suppose that the first starting date in any time-series data set is $\mathbf{t}=0$. Note that some base time series are the direct inputs, whereas some are used to derive another set of time series. 
For instance, the derived time series in our case study are shown in Table 2. The decision parameters used in the case study are defined in Table 3. Let us consider the following constraint $\mathrm{C}$ as an illustration:

$$
\begin{aligned}
& \mathrm{C}\left(\operatorname{SPD}(\mathrm{t}), \mathrm{CG}(\mathrm{t}), \mathrm{CCD}(\mathrm{t}), \operatorname{ISM}(\mathrm{t}), \operatorname{NLCD}(\mathrm{t}), p_{1}, p_{2}, p_{3}, p_{4}, p_{5}\right)= \\
& \operatorname{SPD}(\mathrm{t})<p_{1} \wedge \mathrm{CG}(\mathrm{t})<p_{2} \wedge \mathrm{CCD}(\mathrm{t})<p_{3} \wedge \operatorname{ISM}(\mathrm{t})<p_{4} \wedge \operatorname{NLCD}(\mathrm{t})>p_{5}
\end{aligned}
$$

It means that the parametric monitoring constraint $\boldsymbol{C}$ is satisfied, i.e., its interpretation is True, if the above inequalities with the decision parameters are satisfied at the time point $t$. The interpretation also indicates that the monitoring event occurs. We assume that the investor buys the S\&P 500 index fund at the decision variable time $t$ and sell it at the given $t_{S}$, which is the last day of the given training data set. The earning function $\mathrm{SP}\left(\mathrm{t}_{\mathrm{S}}\right) / \mathrm{SP}(\mathrm{t})-1 \in \mathrm{R}$ is the utility, which is maximized by choosing the optimal value $t \in T$, where $\mathrm{SP}\left(\mathrm{t}_{\mathrm{S}}\right)$ and $\mathrm{SP}(\mathrm{t})$ are the sell and buy value of the S\&P 500 index fund at the time $t_{S}$ and $t$ respectively. The EQPE problem and solution for our example can be constructed by putting the considered time series, parameters, constraints, and functions to the definitions shown in Table 4.

Table 1. Base Time-Series Data.

\begin{tabular}{ll}
\hline Base Time Series S & Abbreviation \\
\hline S\&P 500 & SP(t) \\
Coppock Guide & CG $(\mathrm{t})$ \\
Consumer Confidence & CC(t) \\
ISM Manufacturing Survey & ISM $(\mathrm{t})$ \\
Negative Leadership Composite & NLC $(\mathrm{t})$ \\
\hline
\end{tabular}

Table 2. Derived Time-Series Data.

\begin{tabular}{ll}
\hline Derived Time Series $\mathrm{S}$ & Abbreviation \\
\hline Percentage decline in SP(t) at the time point $\mathrm{t}$ & $\mathrm{SPD}(\mathrm{t})$ \\
$\begin{array}{l}\text { Points drop in } \mathrm{CC}(\mathrm{t}) \text { at the time point } \mathrm{t} \\
\text { Number of consecutive days in Bear Market "DISTRIBUTIOIN" of NLC( } \mathrm{t}) \text { at } \\
\text { and before the time point } \mathrm{t}\end{array}$ & $\mathrm{CCD}(\mathrm{t})$ \\
$\begin{array}{l}\text { Time Utility Earning at the time point } \mathrm{t} \text {, i.e., the index fund is bought at } \mathrm{t} \text { and } \\
\text { sold at } \mathrm{t}_{\mathrm{s}}, \text { where } \mathrm{t}_{\mathrm{s}} \text { is the last day of the learning data set }\end{array}$ & Earning $(\mathrm{t})$ \\
\hline
\end{tabular}

Table 3. Decision Parameters.

\begin{tabular}{ll}
\hline Parameter & Interpretation \\
\hline$p_{1}$ & Test if $\operatorname{SPD}(\mathrm{t})$ is less than $p_{1}$ at $\mathrm{t}$. \\
$p_{2}$ & Test if $\mathrm{CG}(\mathrm{t})$ is less than $p_{2}$ at $\mathrm{t}$. \\
$p_{3}$ & Test if $\mathrm{CCD}(\mathrm{t})$ is less than $p_{3}$ at $\mathrm{t}$. \\
$p_{4}$ & Test if $\operatorname{ISM}(\mathrm{t})$ is less than $p_{4}$ at $\mathrm{t}$. \\
$p_{5}$ & Test if $\mathrm{NLCD}(\mathrm{t})$ is greater than $p_{5}$ at $\mathrm{t}$. \\
\hline
\end{tabular}


Table 4. EQPE Problem and Solution Formulation for S\&P 500 Index Fund.



The values of the optimal decision parameters can be determined by using the learning algorithm, Checkpoint. Before explaining the Checkpoint algorithm in detail, we first review the concept of Dominance.

Definition 7. Dominance $>$ : Given an EQPE problem $\langle\boldsymbol{S}, \boldsymbol{P}, \boldsymbol{C}, \boldsymbol{U}\rangle$ and any two time points $t, t^{\prime} \in \mathrm{T}$, we say that $t^{\prime}$ dominates $t$, denoted by $t^{\prime}>t$, if the following conditions are satisfied:

(1) $0 \leq t^{\prime}<t$, and

(2) $\forall\left(p_{1}, p_{2}, \ldots, p_{n}\right) \in R^{n}, \mathrm{C}\left(S_{l}(t), S_{2}(t), \ldots, S_{k}(t), p_{1}, p_{2}, \ldots, p_{n}\right) \rightarrow \mathrm{C}\left(S_{l}\left(t^{\prime}\right), S_{2}\left(t^{\prime}\right), \ldots\right.$, $\left.S_{k}\left(t^{\prime}\right), p_{1}, p_{2}, \ldots, p_{n}\right)$.

Intuitively, $t^{\prime}$ dominates $t$ if for any selection of parametric values, the query constraint satisfaction at $t$ implies the satisfaction at $t^{\prime}$. Clearly, the dominated time points should be discarded when the optimal time point is being determined. We formally claim that: Claim 1 - Given the conjunctions of inequality constraints, $S_{l}(t)$ $o p_{1} p_{1} \wedge S_{2}(t) o p_{2} p_{2} \wedge \ldots \wedge S_{k}(t) o p_{k} p_{k}$ and the two time points $t^{\prime}, t$ such that $0 \leq t^{\prime}<t$, $t^{\prime}>t$ if and only if $S_{I}\left(t^{\prime}\right) o p_{1} S_{I}(t) \wedge S_{2}\left(t^{\prime}\right) o p_{2} S_{2}(t) \wedge \ldots \wedge S_{k}\left(t^{\prime}\right) o p_{k} S_{k}(t)$. The proof is shown in Appendix.

For example, suppose there are three time series $S_{1}, S_{2}, S_{3}$ and three decision parameters $p_{1}, p_{2}, p_{3}$. And the constraints are $\mathrm{C}\left(S_{1}(t), S_{2}(t), S_{3}(t), p_{1}, p_{2}, p_{3}\right)=S_{1}(t) \geq p_{1}$ $\wedge S_{2}(t) \geq p_{2} \wedge S_{3}(t) \leq p_{3}$. Also assume the values for $S_{1}, S_{2}$, and $S_{3}$ at the time point $t_{1}$, $t_{2}$, and $t_{3}$ respectively in Table 5 shown in the next page.

In this case, the time point $t_{3}$ is dominated because there is a time point $t_{1}$ that make the inequality, $S_{1}\left(t_{1}\right) \geq S_{1}\left(t_{3}\right) \wedge S_{2}\left(t_{1}\right) \geq S_{2}\left(t_{3}\right) \wedge S_{3}\left(t_{1}\right) \leq S_{3}\left(t_{3}\right)$, equal to true.

On the contrary, for all $t^{\prime}<t$, if $S_{l}\left(t^{\prime}\right) \neg o p_{1} S_{I}(t) \vee S_{2}\left(t^{\prime}\right) \neg o p_{2} S_{2}(t) \vee \ldots \vee S_{n}\left(t^{\prime}\right) \neg o p_{n}$ $S_{n}(t)$ is satisfied, $t$ is not dominated by $t^{\prime}$ denoted by $t^{\prime} \ngtr t$. Let us consider the same example above. Because $S_{1}\left(t_{1}\right)<S_{1}\left(t_{2}\right) \vee S_{3}\left(t_{1}\right)>S_{3}\left(t_{2}\right), t_{2}$ is not dominated.

\section{Checkpoint Algorithm and Experimental Evaluation}

Conceptually, we can search a particular set of parameters $\left\{p_{1}, p_{2}, \ldots, p_{n}\right\}$ which is at the earliest time point $t$ that is not dominated by any $t^{\prime}$ such that the value of the objective function $\boldsymbol{O}$ is maximal among all the instantiations of values into parameters. 
However, the problem of this approach is that for every single parameter set at $t$ in a learning data set, the parameter set at $t$ has to be examined with all the previous sets of parameters at $t^{\prime}$ for checking the non-dominance before the optimal solution can be found. In fact, due to the quadratic nature, the conceptual approach is time consuming and expensive particularly if the size of the learning data set is significantly large. Instead, the Checkpoint algorithm uses the KD-tree data structure and searching algorithm $[5,6,7]$ to evaluate whether a time point $t$ is dominated based on the Claim 1 for checking the non-dominance. The pseudo code of the algorithm is:

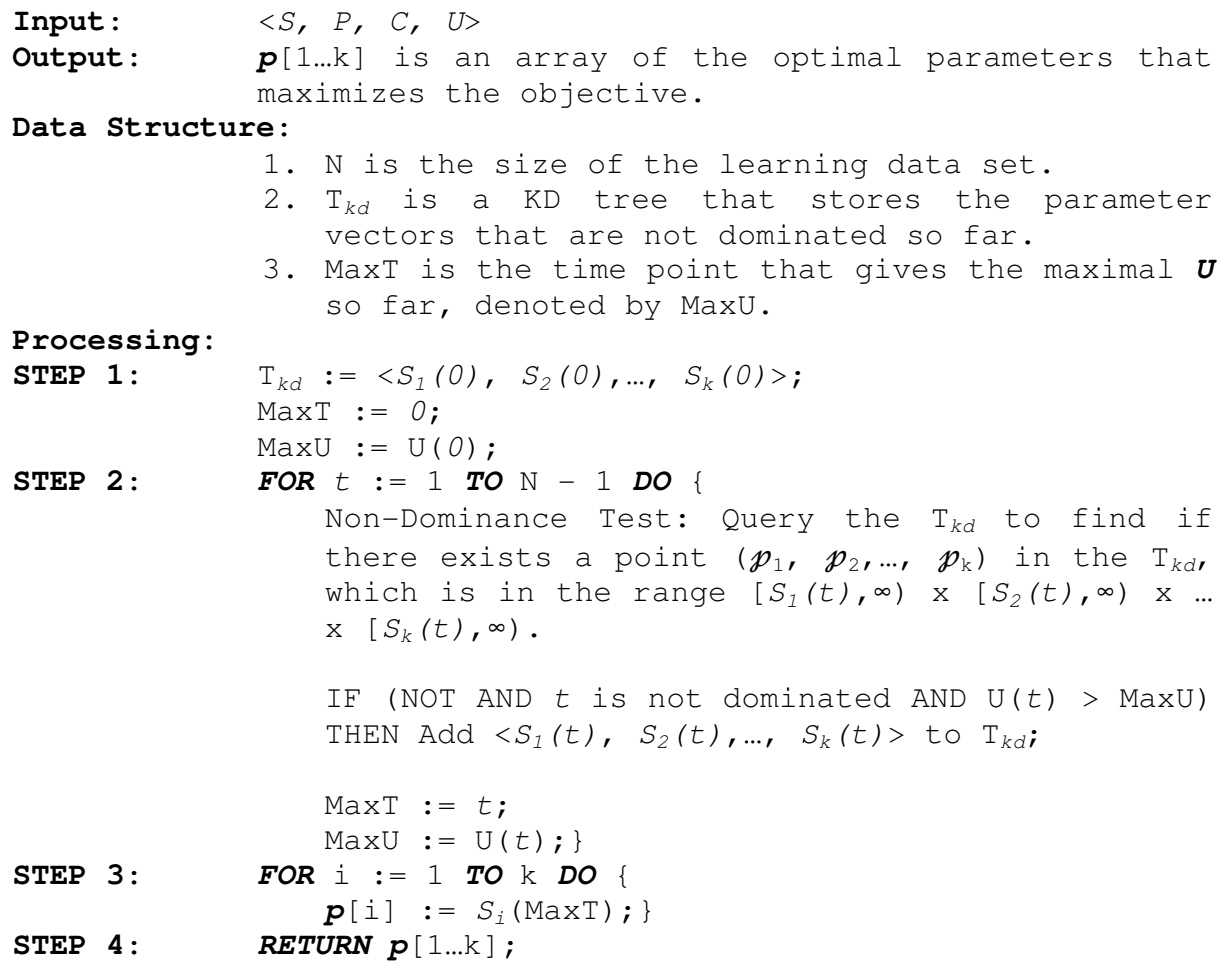

Clearly, the first time point is not dominated because there is no time point preceding it. Therefore, $<S_{l}(0), S_{2}(0), \ldots, S_{k}(0)>$ can be added to $\mathrm{T}_{k d} .0$ and $\mathrm{U}(0)$ can be assigned to MaxT and MaxU respectively.

Using the Checkpoint algorithm step by step for the problem shown in Table 5, we can search through a particular set of parameters $\left\{p_{1}, p_{2}, p_{3}\right\}$ which is at the earliest time point $t$ that is not dominated by any $t^{\prime}$ such that the value of the utility function $\boldsymbol{U}$ is maximal. In STEP 1, the $<S_{1}\left(t_{1}\right), S_{2}\left(t_{1}\right), S_{3}\left(t_{l}\right)>$ is added to the $\mathrm{T}_{k d}$ since it is the first time point. Then $t_{1}$ and $\mathrm{U}\left(t_{1}\right)$ are assigned to MaxT and MaxU respectively. In STEP 2, $t_{2}$ is not dominated because $S_{1}\left(t_{1}\right)<S_{1}\left(t_{2}\right) \wedge S_{2}\left(t_{1}\right)>S_{2}\left(t_{2}\right) \wedge S_{3}\left(t_{1}\right)>S_{3}\left(t_{2}\right)$ does not satisfy the Claim 1 . However, $t_{3}$ is dominated because $S_{l}\left(t_{1}\right)>S_{1}\left(t_{3}\right) \wedge S_{2}\left(t_{1}\right)>S_{2}\left(t_{3}\right) \wedge$ $S_{3}\left(t_{1}\right)<S_{3}\left(t_{3}\right)$ does satisfy the Claim 1. $<S_{1}\left(t_{2}\right), S_{2}\left(t_{2}\right), S_{3}\left(t_{2}\right)>$ is added to the $\mathrm{T}_{k d}$ because $t_{2}$ is not dominated and $\mathrm{U}\left(t_{2}\right)>\mathrm{U}\left(t_{1}\right)$. Thus $t_{2}$ and $\mathrm{U}\left(t_{2}\right)$ are assigned to MaxT and MaxU respectively. In STEP 3, $p[1]:=S_{I}(\operatorname{MaxT}), p[2]:=S_{2}(\operatorname{MaxT})$, and $p[3]:=$ 
$S_{3}(\operatorname{MaxT})$ in the for-loop statement. In STEP 4, the algorithm returns 25, 15, and 2 respectively.

The time complexity for the range search and insertion of a parameter vector in the $\mathrm{T}_{k d}$ tree is $O(k \log N)$ respectively.

Theorem 1: For $N$ parameter vectors in the data set, the Checkpoint algorithm correctly computes an EQPE solution, i.e., $\operatorname{argmax} \boldsymbol{O}\left(p_{1}, p_{2}, p_{3}, p_{4}, p_{5}\right)$, where $\boldsymbol{O}$ is the objective function of the EQPE problem, with the complexity $O(k N \log N)$. The proof of the theorem is shown in Appendix.

Using the Checkpoint algorithm, we can obtain the optimal decision parameters and the maximal earning from the training data set for the financial problem shown in Table 6. The time complexity of the MLE for the logit regression model is $O\left(k^{2} N\right)$, where $k$ is the number of decision parameters, and $\mathrm{N}$ is the size of the learning data set. For the Checkpoint algorithm, the complexity is $O(k N \log N)$. Using the decision parameters from the financial expert (i.e., -20\%, 0, -30, 45, 180 days), the logit regression model, and the Checkpoint algorithm, the "Best Buy" opportunities in stock and their earnings are shown in Table 7. Note that the Checkpoint algorithm considerably outperforms both the financial expert's criteria and the logit regression model.

Table 5. Values of $S_{1}, S_{2}, S_{3}$, and $U$ at the time point $t_{1}, t_{2}$, and $t_{3}$.

\begin{tabular}{lllll}
\hline Time & $\mathrm{S}_{1}$ & $\mathrm{~S}_{2}$ & $\mathrm{~S}_{3}$ & $\mathrm{U}$ \\
\hline $\mathrm{t}_{1}$ & 13 & 27 & 3 & 10 \\
$\mathrm{t}_{2}$ & 25 & 15 & 2 & 200 \\
$\mathrm{t}_{3}$ & 10 & 20 & 5 & 150 \\
\hline
\end{tabular}

Table 6. Optimal Decision Parameters and Maximum Earning (\%) from the Learning Data Set ${ }^{1}$.

\begin{tabular}{llllll}
\hline $\mathrm{p}_{1}$ & $\mathrm{p}_{2}$ & $\mathrm{p}_{3}$ & $\mathrm{p}_{4}$ & $\mathrm{p}_{5}$ & $\mathrm{O}\left(\mathrm{p}_{1}, \mathrm{p}_{2}, \mathrm{p}_{3}, \mathrm{p}_{4}, \mathrm{p}_{5}\right)$ \\
\hline-29.02 & -20.01 & -26.61 & 49 & 70 & 53.37 \\
\hline
\end{tabular}

Table 7. Investors' Earning of the S\&P 500 Index Fund from the Test Data Set ${ }^{2}$.

\begin{tabular}{llll}
\hline Decision Approach & Best Buy & S\&P 500 Index & Earning \% \\
\hline Financial Expert's Criteria & $10 / 09 / 08$ & 909.92 & 1.03 \\
Logit Regression Model & $11 / 26 / 08$ & 887.68 & 3.56 \\
$\begin{array}{l}\text { Checkpoint Algorithm with Financial Expert's } \\
\text { Template }\end{array}$ & $03 / 10 / 09$ & 719.6 & 27.8 \\
\hline
\end{tabular}

1 The learning data set is from $06 / 01 / 1997$ to $06 / 30 / 2005$.

2 The test data set is from $07 / 01 / 2005$ to $06 / 30 / 2009$ that is the sell date of the fund with the value of 919.32 . 


\section{MTSA Data Model and Query Language}

\subsection{Data Model}

The time-series (TS) data model is an extension of the relational database model with specialized schemas. A time-series schema is of the form TSname(T:Time, Vname:Vtype), where Time and Vtype are data types, Vtype is either Real or Integer, and TSname and Vname are names chosen by users.

A time-event (TE) schema is of the form TEname(T:Time, Ename:Binary), where Binary is the binary type corresponding to the domain $\{0,1\}$, and TEname and Ename are names chosen by users.

A TS database schema is a set of relational schemas which may include (specific) TS and/or TE schemas.

A TS tuple over a schema TSname(T:Time, Vname:Vtype) is a relational tuple over that schema, i.e., a mapping $\mathrm{m}:\{\mathrm{T}$, Vname $\} \rightarrow$ Dom(Time) x Dom(Vtype), such that $\mathrm{m}(\mathrm{T}) \in \operatorname{Dom}($ Time) and $\mathrm{m}($ Vname $) \in \operatorname{Dom}($ Vtype).

A TE tuple over a similar schema TEname(T:Time, Ename:Binary) is a mapping $\mathrm{m}:\{\mathrm{T}$, Ename $\} \rightarrow$ Dom(Time) $\mathrm{x}$ Dom(Binary), such that $\mathrm{m}(\mathrm{T}) \in \operatorname{Dom}($ Time) and $\mathrm{m}($ Ename $) \in \operatorname{Dom}$ (Binary).

Let us consider our financial example. In the market-bottom scenario, the service provider can use the querying service to create the base, derived, and related timeseries tables as inputs and store them in the database. The base time-series tables are SP(T, Index), CG(T, Index), CC(T, Index), ISM(T, Index), and NLC(T, Index).

\subsection{Querying Service}

Using the base time series tables, we can generate derived time series tables (if any) by the traditional SQLs. In our case study, some derived time series tables, e.g., $\operatorname{SPD}(\mathrm{t}), \mathrm{CCD}(\mathrm{t})$, etc., are:






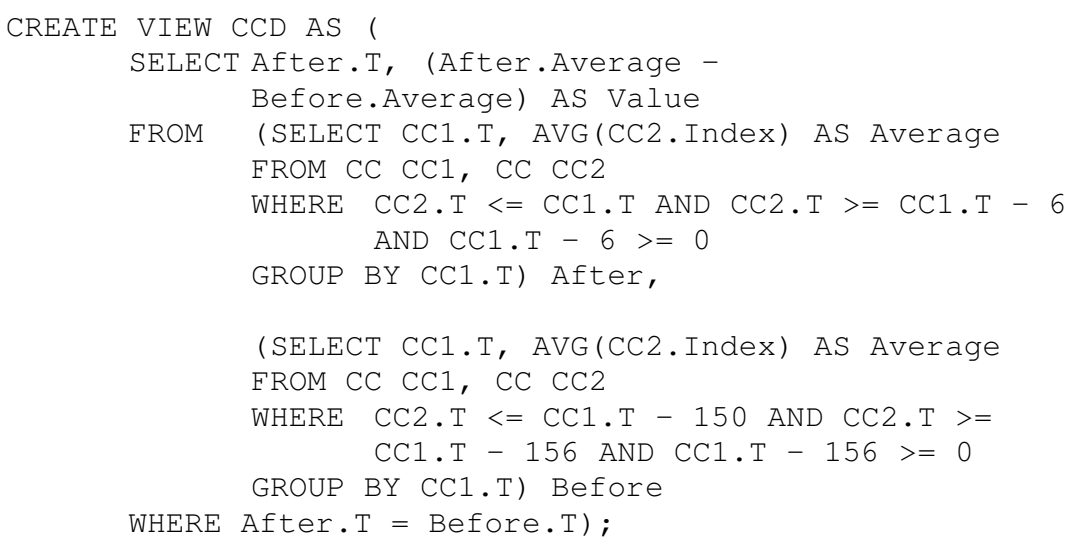

\subsection{Monitoring and Recommendation Service}

Using the monitoring and recommendation service over the new incoming data, the financial analyst can recommend the investors whether or not they should buy the stock. In our example, the input parametric time series tables for monitoring are SPD(T, Value), CG(T, Index), CCD(T, Value), ISM(T, Index), and NLCD(T, Value). The monitoring and recommendation service can be expressed by a monitoring view and executed by the MONITOR command.

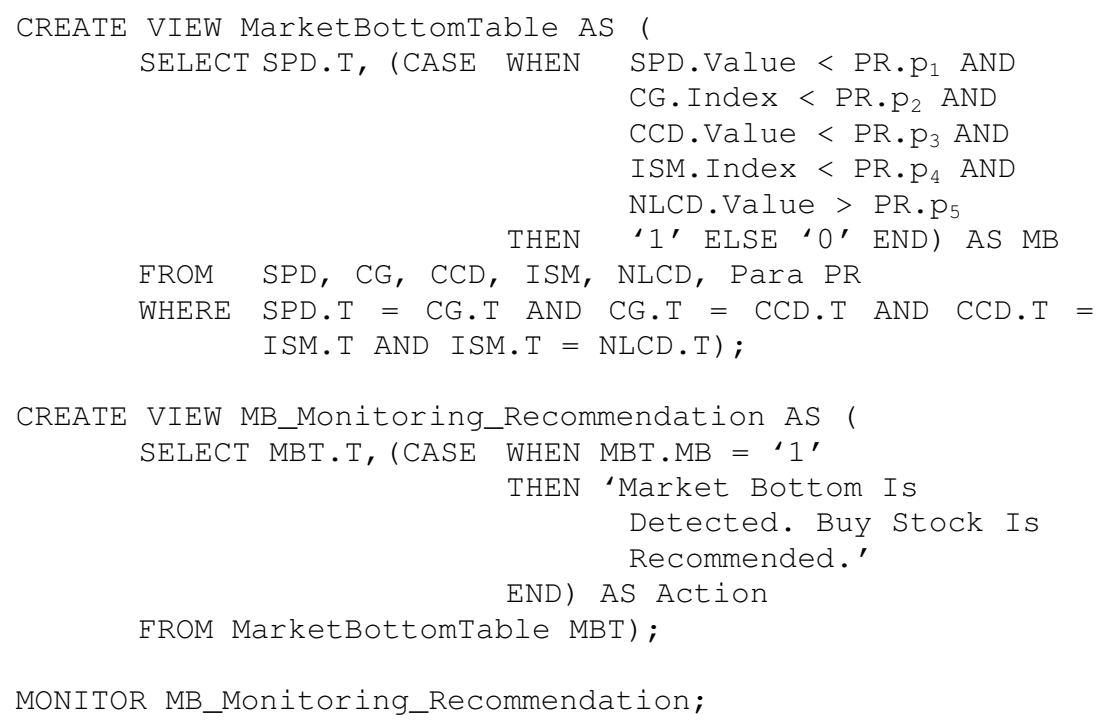

,where Para is a table to store the decision parameters, e.g., $\mathrm{p}_{1}=-20, \mathrm{p}_{2}=0, \mathrm{p}_{3}=-$ $30, \mathrm{p}_{4}=45$, and $\mathrm{p}_{5}=180$. If the parametric monitoring constraint in the "CASE WHEN" clause is satisfied at the current time point $t$, the value of the attribute "MB" 
indicates " 1 ". The service then recommends the financial analysts to buy the index fund for the investors since the market bottom is predicted.

\subsection{Parameter Learning Service}

As we discussed, the expert's suggested parameters $(-20.0,-30,45,180)$ are not accurate enough to monitor the dynamic financial market at all time; thus, the parameter learning service should be adopted by expressing as follows:

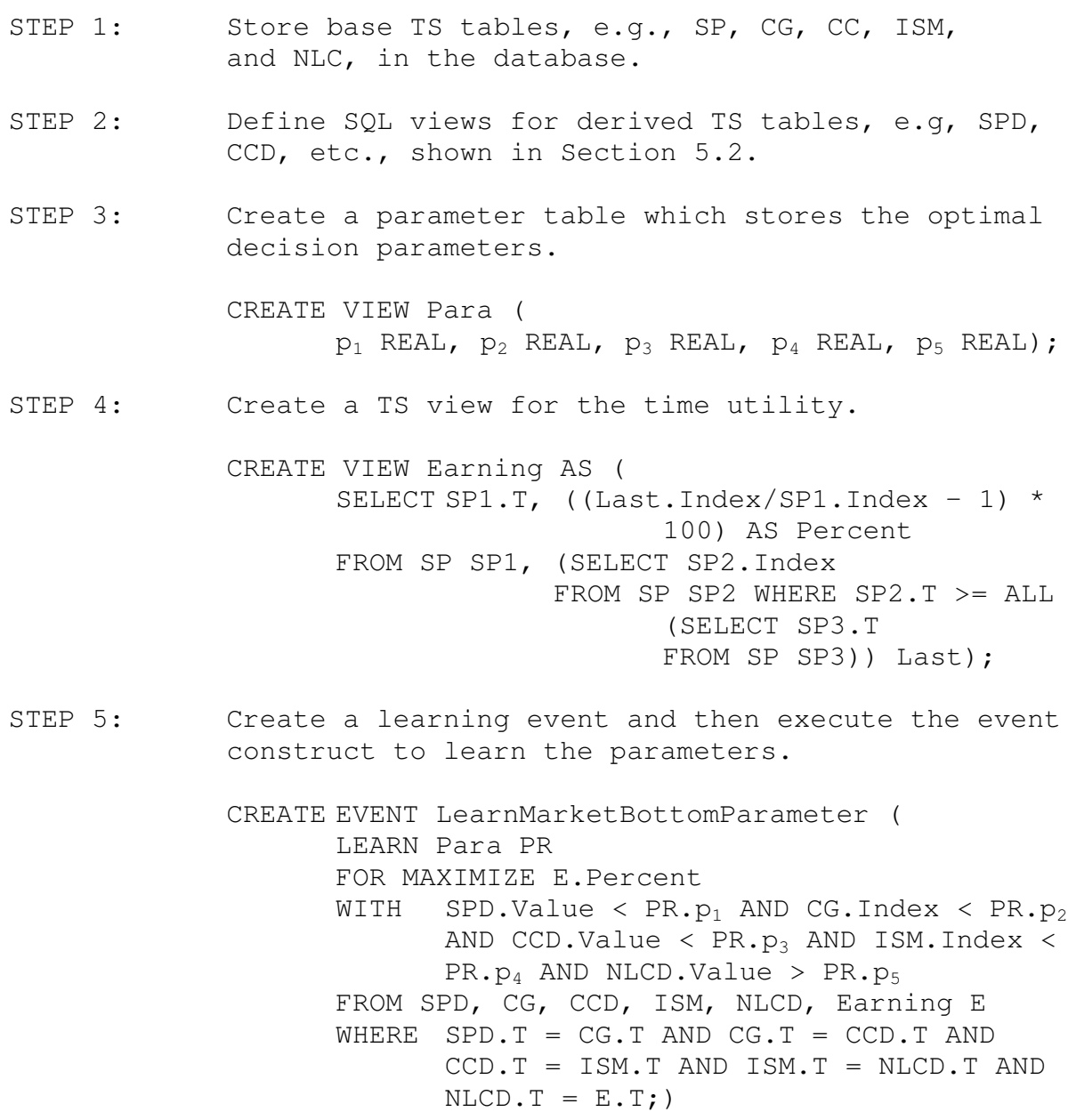

EXECUTE LearnMarketBottomParameter;

When the event "LearnMarketBottomParameter" is executed, the command "LEARN" will call for the Checkpoint algorithm to solve the corresponding EQPE problem and will put its solution in the Para table, where all parameters, e.g., $\mathrm{p}_{1}, \mathrm{p}_{2}, \mathrm{p}_{3}$, $\mathrm{p}_{4}$, and $\mathrm{p}_{5}$ are instantiated with optimal values. 


\section{Conclusions and Future Work}

To the best of our knowledge, this is the first paper to propose an event-based service framework for multivariate time series analytics that provides model definition, querying, parameter learning, model evaluation, monitoring, and decision recommendation on events over multivariate time series. The parameter learning services combine the strengths of both domain-knowledge-based and formal-learningbased approaches for maximizing utility on events over the time series. It includes a mathematical model and a learning algorithm for solving Expert Query Parametric Estimation problems. Using the framework, we conduct a preliminary experiment in the financial domain to demonstrate that our model and algorithm are more effective and produce results that are superior to the two approaches mentioned above. We also develop MTSA data model and query language for the services of querying, monitoring, and parameter learning. There are still many open research questions, e.g., which model can capture and integrate multiple, inter-related events, what algorithm can simultaneously learn decision parameters optimally for those events, and how those events will impact the services that the framework provides.

\section{References}

1. Stack, J.B., 2009. Technical and Monetary Investment Analysis, Vol 9 Issue 3 \& 5. InvesTech Research.

2. Dougherty, C., 2007. Introduction to Econometrics (Third Edition). Oxford University Press.

3. Hansen, B.E., 2010. Econometrics. University of Wisconsin. http://www.ssc.wisc.edu/ bhansen/econometrics/Econometrics.pdf.

4. Heij, D., De Boer, P., Franses, P.H., Kloek, T., and Van Dijk, H.K., 2004. Econometric Methods with Applications in Business and Economics. Oxford University Press.

5. Bentley, J.L., 1975. Multidimensional Binary Search Trees Used for Associative Searching. Communications of the ACM, Vol 18 Issue 09, p. 509-517, 1975.

6. Bentley, J.L., 1979. Multidimensional Binary Search Trees in Database Applications, Vol 5 Issue 04, p. 333-340. IEEE Transactions on Software Engineering.

7. Samet, H., 2006. Foundations of Multidimensional and Metric Data Structures. Morgan Kaufmann.

8. Bellman, R., 1961. Adaptive Control Processes: A Guided Tour. Princeton, University Press.

9. Brodsky, A., Bhot, M.M., Chandrashekar, M., Egge, N.E., and Wang, X.S., 2009. A Decisions Query Language (DQL): High-Level Abstraction for Mathematical Programming over Databases. Proceedings of the 35th SIGMOD International Conference on Management of Data.

10. Brodsky, A., Henshaw, S.M., and Whittle, J., 2008. CARD: A Decision-Guidance Framework and Application for Recommending Composite Alternatives. 2nd ACM International Conference on Recommender Systems.

11. Brodsky, A. and Wang. X.S., 2008. Decision-Guidance Management Systems (DGMS): Seamless Integration of Data Acquisition, Learning, Prediction, and Optimization. Proceedings of the 41st Hawaii International Conference on System Sciences. 
12. Dumas, M., O’Sullivan, J., Heravizadeh, M., Edmond, D., and Hofstede, A., 2001. Towards a Semantic Framework for Service Description. Proceedings of the IFIP TC2/WG2.6 Ninth Working Conference on Database Semantics: Semantic Issues in E-Commerce Systems.

13. Erl, T., 2005. Service-Oriented Architecture (SOA): Concepts, Technology, and Design. Prentice Hall.

14. Erradi, A., Anand, S., and Kulkarni, N., 2006. SOAF: An Architectural Framework for Service Definition and Realization. IEEE International Conference on Services Computing (SCC'06).

15. Harrington, J., 2009. Relational Database Design and Implementation (Third Edition). Morgan Kaufmann.

16. Holyfield, S., 2005. Non-technical Guide to Technical Frameworks. JISC CETIS. http://www.elearning.ac.uk/features/nontechguide1.

17. Josuttis, N., 2007. SOA in Practice: The Art of Distributed System Design. O'Reilly Media.

18. Ngan, C.K., Brodsky, A., and Lin, J., 2010. Decisions on Multivariate Time Series: Combining Domain Knowledge with Utility Maximization. The 15th IFIP WG8.3 International Conference on Decision Support Systems.

19. Nicholls, P., 2009. Enterprise Architectures and the International e-Framework. eframework Organization. http://www.e-framework.org/Portals/9/docs/EAPaper_200907.pdf

20. Olivier, B., Roberts, T., and Blinco, K., 2005. The e-Framework for Education and Research: An Overview. e-framework Organization. http://www.eframework.org/Portals/9/Resources/eframeworkrV1.pdf.

21. Ort, Ed. Service-Oriented Architecture and Web Services: Concepts, Technologies, and Tools. Sun Developer Network Technical Articles and Tips. http://java.sun.com/developer/technicalArticles/WebServices/soa2/

22. Papazoglou, M., and Heuvel, W., 2005. Service Oriented Architectures: Approaches, Technologies, and Research Issues. The VLDB Journal, June.

23. Quartel, D., Steen, M., Pokraev S., and Sinderen, M., 2007. COSMO: A Conceptual Framework for Service Modelling and Refinement, Volume 9, Numbers 2-3, 225-244, July. Journal of Information Systems Frontiers.

24. Ralph H. Sprague, Jr., 1980. A Framework for the Development of Decision Support Systems, Volume 4, Number 4, 1-26, December. MIS Quarterly.

25. Stephen, B., et al., 2008. Database Design: Know It All. Morgan Kaufmann.

26. Wilson, S., Blinco, K., and Rehak, D., 2004. Service-Oriented Frameworks: Modelling the Infrastructure for the Next Generation of e-Learning Systems. JISC CETIS. http://www.jisc.ac.uk/uploaded_documents/AltilabServiceOrientedFrameworks.pdf.

27. Zhang, T., Ying, S., and Cao, S., and Jia, S., 2006. A Modeling Framework for ServiceOriented Architecture. Proceedings of the Sixth International Conference on Quality Software (QSIC'06).

\section{Appendix}

Claim 1 - Given the conjunctions of inequality constraints, $S_{l}(t) o p_{1} p_{1} \wedge S_{2}(t) o p_{2} p_{2} \wedge \ldots$ $\wedge S_{k}(t) o p_{k} p_{k}$ and the two time points $t^{\prime}, t$ such that $0 \leq t^{\prime}<t, t^{\prime}>t$ if and only if $S_{I}\left(t^{\prime}\right) o p_{I}$ $S_{1}(t) \wedge S_{2}\left(t^{\prime}\right) o p_{2} S_{2}(t) \wedge \ldots \wedge S_{k}\left(t^{\prime}\right) o p_{k} S_{k}(t)$.

\section{Proof:}

Without loss of generality, we assume that $o p_{i}=$ " $\leq$ ", for all $1 \leq \mathrm{i} \leq \mathrm{k}$. It is because " $\geq$ " can be replaced with $\leq$ by changing a corresponding time series $S_{i}(t)$ to $-S_{i}(t)$. For $o p_{i}=$ "=", we can use the conjunction with both $\geq$ and $\leq$. 
If Direction:

Assume that $S_{l}\left(t^{\prime}\right) \leq S_{l}(t) \wedge S_{2}\left(t^{\prime}\right) \leq S_{2}(t) \wedge \ldots \wedge S_{k}\left(t^{\prime}\right) \leq S_{k}(t)$. For any $\left(p_{l}, p_{2}, \ldots, p_{k}\right) \in \mathrm{R}^{\mathrm{k}}$ and every $\mathrm{i}=1,2, \ldots, \mathrm{k}$, if $S_{I}(t) \leq p_{1}$, then $S_{I}\left(t^{\prime}\right) \leq p_{I}$ because $S_{I}\left(t^{\prime}\right) \leq S_{I}(t)$. Therefore, $S_{l}(t)$ $\leq p_{1} \wedge S_{2}(t) \leq p_{2} \wedge \ldots \wedge S_{k}(t) \leq p_{k} \rightarrow S_{I}\left(t^{\prime}\right) \leq p_{1} \wedge S_{2}\left(t^{\prime}\right) \leq p_{2} \wedge \ldots \wedge S_{k}\left(t^{\prime}\right) \leq p_{k}$ and then $t^{\prime}>t$.

Only If Direction:

Assume that $t^{\prime}>t$. Then $S_{I}(t) \leq p_{1} \wedge S_{2}(t) \leq p_{2} \wedge \ldots \wedge S_{k}(t) \leq p_{k} \rightarrow S_{l}\left(t^{\prime}\right) \leq p_{1} \wedge S_{2}\left(t^{\prime}\right) \leq p_{2}$ $\wedge \ldots \wedge S_{k}\left(t^{\prime}\right) \leq p_{k}$. Therefore, for any $\left(p_{1}, p_{2}, \ldots, p_{k}\right) \in \mathrm{R}^{\mathrm{k}}$ and every $\mathrm{i}=1,2, \ldots, \mathrm{k}$, we have $S_{i}(t) \leq p_{i} \rightarrow S_{i}\left(t^{\prime}\right) \leq p_{i}$

Proof of Theorem 1: The Checkpoint algorithm correctly solves the EQPE problem, i.e., if $\operatorname{argmax} \boldsymbol{O}\left(p_{1}, p_{2}, p_{3}, p_{4}, p_{5}\right)$, where $\boldsymbol{O}$ is the objective function of the EQPE problem.

The time complexity is $O(k N \log N)$, where k is the number of time series and $\mathrm{N}$ is the size of the learning data set.

Proof: To prove the correctness of the algorithm if it is sufficient to show Claim 2: The Non-Dominance test in STEP 2 of the Checkpoint Algorithm is satisfied at the time point $\mathrm{t}$ if and only if there does not exist $t^{\prime}$ that dominates $t$, where $0 \leq t^{\prime}<t$.

We prove it by induction on $t$, where $1 \leq t \leq \mathrm{N}$. For $t=1, \mathrm{~T}_{k d}=\emptyset$, and $t=1$ is not dominated; therefore, the "if and only if" condition holds. Assuming the correctness for 1 , $2, \ldots, t-1$; it follows the STEP 2 of the algorithm that $\mathrm{T}_{k d}$ at the point $t$ contains all the non-dominated time points $t^{\prime}$, where $t^{\prime} \leq t-1$.

\section{If Direction:}

The IF part of the Claim 2 is straightforward since if $t$ is not dominated by an earlier time point $t^{\prime}$, such point cannot appear on the $T_{k d}$ tree; therefore, the Non-Dominance Test must be satisfied by the Claim 1 .

Only If Direction:

For the ONLY IF part of the Claim 2, assume that the Non-Dominance test in STEP 2 of the algorithm is satisfied. Then there does not exist the time point $t^{\prime}$ on $\mathrm{T}_{k d}$ for which $\left(S_{I}\left(t^{\prime}\right)\right.$, $\left.S_{2}\left(t^{\prime}\right), \ldots, S_{k}\left(t^{\prime}\right)\right) \in\left[S_{I}(t), \infty\right) \times\left[S_{2}(t), \infty\right) \times \ldots \times\left[S_{k}(t), \infty\right)$, where $0 \leq t^{\prime}<t$. Assume that $\mathrm{T}_{k d}$ at the time $t$ contains the time points $t_{1_{i}}<t_{2_{i}}<\cdots<t_{m_{i}}$ and assume, by contradiction, that there exists $t^{\prime}$ that dominates $t, t^{\prime}>t$, where $0 \leq t^{\prime}<t$. Clearly, $t^{\prime}$ is not one of $t_{1_{i}}<t_{2_{i}}<$ $\cdots<t_{m_{i}}$ because they do not dominate $t$ by the induction hypothesis. Because $t^{\prime}$ was not added to the $\mathrm{T}_{k d}$ tree and the induction hypothesis, $t_{j_{i}}>t^{\prime}$ for some $\mathrm{j}=1,2, \ldots, \mathrm{m}$. From the contradiction assumption $t^{\prime}>t$ and the transitivity of $>$, it follows that $t_{j_{i}}>t$. Thus, by the Claim 1, $S_{1}\left(t_{j_{i}}\right) \leq S_{1}(t) \wedge S_{2}\left(t_{j_{i}}\right) \leq S_{2}(t) \wedge \cdots \wedge S_{k}\left(t_{j_{i}}\right) \leq S_{k}(t)$ which contradicts the fact that the Non-dominance test in STEP 2 was satisfied for $t$. This completes the proof of the Claim 2 and of the correctness of the algorithm.

Time Complexity: The algorithm performs $\mathrm{N}$ iterations in STEP 2, spending time $O(k \log N)$ using the $\mathrm{T}_{k d}$ algorithm for the $\mathrm{T}_{k d}$ range query in Non-Dominance Test. Thus the overall complexity is $O(k N \log N)$. 